\title{
CLINICAL PROFILE AND MANAGEMENT OF EMPYEMEYA THORACIS: EXPERIENCE FROM EASTERN NEPAL
}

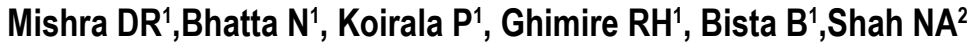 \\ ${ }^{1}$ Division of Pulmonary, Critical Care \& Sleep Medicine, Department of Internal Medicine, B.P. Kiorala Institute of \\ Health Sciences, Dharan \\ 2 Department of Pathology, B.P. Kiorala Institute of Health Sciences, Dharan
}

\begin{abstract}
Introduction: The management of Empyema Thoracis is challenging. It requires specialist medical and surgical care at the same time. There are of lack of data regarding the clinical profile and the steps of day to day management, hence this study aims to identify these parameters and focus on the gaps in management that is commonplace in our setting and that are representative of other resource limited settings as well.

Methods: Clinical profile, etiological agents, hospital course and outcome of 30 patients with empyema thoracis treated from 2012 to 2014 in B.P. Kiorala Institute of Health Sciences was analyzed. All patients were diagnosed on the basis of aspiration of frank pus from pleural cavity.
\end{abstract}

Results: 28 cases (93.3\%) were Male and the mean age was $42.07 \pm 18.28$ years. $73.3 \%$ of the empyema was Right sided and $60 \%$ were classified as medium sized and $40 \%$ as small sized. $60 \%$ of the patients were smokers. $80 \%$ of the case were diagnosed as bacterial infection whereas $20 \%$ were presumed tubercular on clinical basis and responded to treatment. Fever was the commonest presentation in $80 \%$ of the cases followed by shortness of breath $(66.7 \%)$, cough $(60 \%)$, chest pain $(53.3 \%)$ and sputum production $(20 \%)$. On investigation, $\mathrm{pH}$ was not measured in the fear of clogging the ABG machine. Pleural fluid glucose was below $40 \mathrm{mg} / \mathrm{dl}$ in all the cases. The median ADA value was 54.30 (15-350) and ADA was higher than $40 \mathrm{U} / \mathrm{l}$ in 10 cases with non-tubercular empyema. All cases were managed with Chest tube insertion and antibiotics. Four patients had to be referred for BPF closure whereas five for decortications. In all cases requiring decortications, a "pleural peel" could be identified in Chest Xrays. Since the patients were being managed in different units the outcome of referred patients could not be ascertained.

Conclusion The gaps in the management of empyema in resource limited setting starts from inappropriate early treatment, inadequate diagnostic facilities, delayed referral and lack of early and appropriate surgical intervention. All these factors combine to the increased morbidity and mortality associated with the management of Empyema thoracis.

Key words: Empyema thoracis, Clinical Profile, Referral

\section{INTRODUCTION}

Empyema refers to the presence of pus in the pleural cavity. Though different cut off levels of

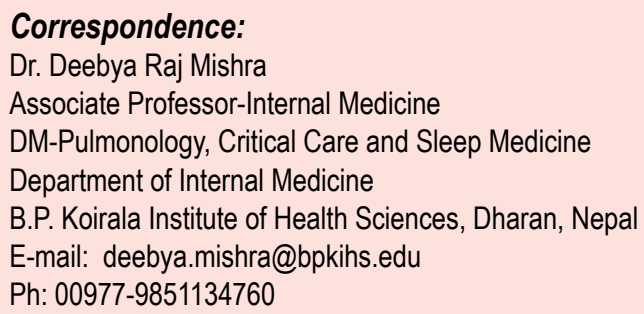

WBC and protein have been used in literature in an attempt to define Empyema ${ }^{1,2}$, the simplest approach is to reserve the term for patients presenting with pleural effusion who have thick, purulent appearing pleural fluid ${ }^{3}$.

The management of Empyema involves antibiotics, tube thoracostomy and often, surgical intervention. It requires specialist Medical and Surgical Care at the same time. As early drainage of pus is essential for the treatment of empyema, surgical procedures besides tube thoracostomy need to be employed early to prevent significant morbidity and mortality. Video Assisted Thoracoscopy Surgery (VATS) is 
the procedure of choice for surgical intervention due to its safety and tolerability ${ }^{4}$. Where VATS is not available, procedures such as rib resection and "window" creation for open drainage are still being employed.

In a resource limited setting without VATS, thoracoscopy or dedicated thoracic surgeon, the only modalities of management remain medical and tube thoracostomy supplemented by intrapleural fibrinolytics. Thus, the morbidity in such setting is expected to be higher and also representative of the prevalent situation in other resource constrained settings.

\section{MATERIALS AND METHODS}

We conducted a retrospective study of the medical records and chest radiographs of consecutive cases diagnosed as Empyema and admitted to the Respiratory and Internal Medicine services of the B P Koirala Institute of Health Sciences, Nepal during a 2 year period from Jan 2012 to Jan 2014.

The hospital serves as a referral hospital for the whole of Eastern Nepal.

In the cases with Empyema, the relevant data were scanned and included in the study only if the patient chart was viewed as Complete. Complete chart was defined as having detailed epidemiological data, a complete medical history and clinical examination, investigations including Pleural fluid analysis for sugar/protein/light's criteria, Adenosine Deaminase and malignant cytology if diagnosis was inconclusive by routine analysis, other special tests of pleural fluid as required, like, Amylase etc. Only the initial fluid examination was recorded. Once the above parameters were present, the Digital Radiographic Library was explored. The X-Ray was reviewed by a Pulmonology trainee. The size of the effusion was estimated on the initial upright inspiratory posteroanterior chest film. Effusions were classified as; large effusion if effusion covered greater than 50 percent of the hemithorax, medium if 25 to 50 percent was covered, and small effusion if effusion covered less than 25 percent of the hemithorax.

\section{STATISTICAL ANALYSIS}

Data with parametric distribution have been expressed in mean \pm SD whereas data with non parametric distribution have been expressed as median (25th percentile and 75th percentile). Data was entered in Microsoft Excel, copied to SPSS 17 and descriptive and frequency data were analyzed.

\section{RESULTS}

A total of 30 cases diagnosed as Empyema had complete medical chart and were considered. 28 cases (93.3\%) were Male and the mean age was $42.07 \pm 18.28$ years. $73.3 \%$ of the Empyema was Right sided and $60 \%$ were classified as Medium sized and $40 \%$ as Small sized. Smoking was the commonest risk factor in $60 \%$ of the cases. $24 / 30(80 \%)$ of the cases were diagnosed as Parapneumonic, whereas $6 / 30(20 \%)$ were diagnosed as Tubercular. Of the cases of Tuberuclar Empyema, AFB staining was positive in two cases whereas the other four cases were diagnosed on clinic-epidemiological background and high ADA levels.

The mean duration of symptoms before presentation to hospital was 23 days. $90 \%$ (27/30) of the patients has already received oral antibiotics from pharmacies.

Fever was the commonest presentation in $80 \%$ of the cases followed by shortness of breath $(66.7 \%)$, cough $(60 \%)$, chest pain $(53.3 \%)$ and sputum production (20\%) (Table 1).

\begin{tabular}{|l|c|}
\hline \multicolumn{2}{|l|}{ Table 1. Symptoms } \\
\hline Symptoms & Frequency (\%) \\
\hline Shortness of breath & $20 / 30(67 \%)$ \\
\hline Fever & $24 / 30(80 \%)$ \\
\hline Cough & $18 / 30(60 \%)$ \\
\hline Sputum production & $6 / 30(20 \%)$ \\
\hline Chest pain & $16 / 30(54 \%)$ \\
\hline Weight loss & $2 / 30(7 \%)$ \\
\hline
\end{tabular}

Six patients were afebrile during presentation. The mean duration of fever in the Tubercular Empyema group was 50 days.

Pleural fluid glucose was below $40 \mathrm{mg} / \mathrm{dl}$ in all the cases. The median ADA value was 54.30(27-141 $\mathrm{U} / \mathrm{l}$ and $\mathrm{ADA}$ was higher than $40 \mathrm{U} / \mathrm{l}$ in 10 cases with non-tubercular empyema (Table 2).

\begin{tabular}{|l|c|}
\hline \multicolumn{2}{|l|}{ Table 2. Pleural fluid features } \\
\hline Parameters & Values \\
\hline Pleural fluid Neutrophils* & $77.33 \pm 25.06$ \\
\hline
\end{tabular}




\begin{tabular}{|l|c|}
\hline Pleural fluid Lymphocytes\# & $10(10-30)$ \\
\hline Pleural fluid Sugar $(\mathrm{mg} / \mathrm{dl})^{*}$ & $14.40 \pm 5.04$ \\
\hline Pleural fluid Protein $(\mathrm{g} / \mathrm{dl})^{*}$ & $4.99 \pm 2.06$ \\
\hline Pleural fluid ADA (U/I)\# & $54.30(27-141)$ \\
\hline
\end{tabular}

*Mean \pm Standard Deviation, \#Median (Interquartile Range, ADA Adenosine Deaminase

Microbiological culture was positive in $11 / 30(37 \%)$ of the cases with 14 organisms being cultured. Staphylococcus was the most common organism (Table3). Anaerobic culture was not employed and Mycobacterial culture was unavailable at the time.

Table 3. Isolation of microorganisms ( $n=30,14$ isolates from 11 patients)

\begin{tabular}{|l|c|}
\hline Organism & Frequency (\%) \\
\hline Staphylococcus aureus & $8 / 14(57 \%)$ \\
\hline Gram negative rods & $5 / 14(36 \%)$ \\
\hline Pneumococcus & $1 / 14(7 \%)$ \\
\hline Anaerobes & $0 / 14$ \\
\hline
\end{tabular}

All cases were managed with Chest tube insertion and antibiotics. 4 patients had to be referred for BPF closure whereas 5 for Decortication. In all cases requiring decortications, a "pleural peel" could be identified in Chest X-rays (Table 4).

\begin{tabular}{|l|c|}
\hline \multicolumn{2}{|l|}{ Table 4. Outcomes. } \\
\hline Outcome Variable & Frequency (\%) \\
\hline Improved & $19 / 30(63 \%)$ \\
\hline Died & $1 / 30(3 \%)$ \\
\hline $\begin{array}{l}\text { Leave Against Medical } \\
\text { Advice }\end{array}$ & $1 / 30(3 \%)$ \\
\hline $\begin{array}{l}\text { Referred for Bronchopleural } \\
\text { fistula closure }\end{array}$ & $4 / 30(14 \%)$ \\
\hline Referred for Decortication & $5 / 30(17 \%)$ \\
\hline
\end{tabular}

\section{DISCUSSION}

The management of Empyema in resource limited setting is punctuated by a lot of gaps.

The mean duration of symptoms before presentation to hospital was 23 days. $90 \%$ of the patients had already received oral antibiotics from pharmacies. The widespread use of antibiotics in the community for even the most trivial of causes leads to decrease in Culture positivity once the patient reaches the hospital. In our study, culture was positive in only $37 \%{ }^{11}$. Again due to resource limitation anaerobe culture is not routine and we speculate this is the same for most settings in our part of the world. At the time of the study, even culture facilities for Mycobacterium were not available. Banga et al. ${ }^{5}$ reported a culture positivity of $42 \%{ }^{13}$ while Baranwal et al. ${ }^{6}$ reported a culture positivity of $48 \%$ in pediatric patients in the same center as ours. Staphylococcus aureus was the commonest organism isolated, a trend found in most other studies as well ${ }^{5,6}$.

Empyema was diagnosed to be Tubercular in $20 \%{ }^{6}$. The diagnosis was on the basis of pus AFB positivity in two cases whereas in the other four cases the diagnosis was made on the basis of clinic-epidemiological background and radiological features. Tuberculosis as a cause of Empyema has been variably reported with rates varying from $0 \%$ to $42 \%$. The varying rates could depend on culture availability, the use of solid or liquid cultures as well as the technique of culture.

The Median pleural fluid ADA value was $54.30 \mathrm{U} / \mathrm{l}$ (IQR,27-141). ADA was more than 40U/I in $42 \%{ }^{10}$ of the cases of Non-Tubercular Empyema. This underlines the difficulty in using ADA values to differentiate the etiology of pleural effusion as Tubercular or Non-Tubercular in cases of Empyema ${ }^{3}$.

Management of the patients involved use of Antibiotics, tube thoracostomy and intrapleural Streptokinase was used in seven cases. Tube thoracostomy was done with $28 \mathrm{Fr}$ chest tube in $60 \%$ cases whereas $14 \mathrm{Fr}$ Pigtail catheters was used in the remaining 12 cases. The distinction was made on appearance and thickness of the pus aspirated. Medical management was successful in $63 \%$ patients. One patient died during treatment and one patient left against medical advice.

Bronchopleual fistula was encountered in $14 \%{ }^{4}$. These patients were observed for two weeks duration after which they were referred for Surgical management. There was no spontaneous improvement in any of the cases. Bronchopleural fistula (BFP) which is an abnormal connection between the bronchus and the pleural space can be divided into central BFP and the peripheral BPF. In the central type, there is direct communication between a large bronchus and the pleural space, whereas in the peripheral type, the air leak is from a peripheral bronchus or from the lung parenchyma 
into the pleural space ${ }^{7,8}$. The common causes of peripheral BPF are necrotising infections such as pulmonary tuberculosis, pneumonia and even empyema ${ }^{8,9}$. The incidence of BPF resulting from these suppurative lung and pleural diseases has not been clearly documented in the literature; however, the incidence following pulmonary or lobar resection ranges between $4-20 \%{ }^{10}$. The management of this condition is very challenging as recurrences are common leading to incomplete removal of the pus; failure of closure of the fistula and inability to obliterate the residual pleural cavity ${ }^{11}$. There was a high rate of BPF in our study compared to other studies. The high rate could be attributed to delayed presentation as well as concomitant pulmonary processes. The presence of BPF complicates the management of Empyema and whether early surgical intervention would be able to reduce the frequency of BPF would be a point of interest. $17 \%{ }^{5}$ had to be referred for Decortications and in all these cases pleural peel was evident on radiological examination. In a study involving 179 patients, Decortications was necessary in $55 \%$ of patients with anaerobic infections and in $50 \%$ with aerobic infections ${ }^{12}$. In another study, decortications was required in $24 \%{ }^{11}$ cases $^{13}$. Surgical decortications has persistently shown to be highly successful in treating Empyema $^{13}$. Wong and colleagues ${ }^{14}$ reported that surgical decortications with VATS significantly reduced hospitalization duration compared with tube drainage treatment in paediatric patients. Thourani and colleagues ${ }^{15}$ reported that surgical decortications reduced the hospital stay compared with simple drainage through a catheter or chest tube. They also calculated that early decortications has charges similar to those of primary intervention (image-directed catheter or tube thoracostomy) but is more cost-effective than failed imagedirected catheter and thus advocated the use of early surgical intervention as the most optimal and cost-effective initial modality for the treatment of empyema thoraces. Though early surgical intervention has become the treatment of choice, how early is early enough is the big question? Chung and colleagues ${ }^{16}$ found that Patients with symptom durations of less than 4 weeks showed better early results than those with symptom durations greater than 4 weeks. Thus, symptom duration can be considered a reliable preoperative factor in deciding the surgical management of empyema or cases involving loculated pleural effusion. The evidence in favor of early surgical management points to a huge resource gap in the management of Empyema in resource limited setting. Not only is the surgical management not done in most centres, referral is also delayed. In our study, the patients were referred after a mean of 43 days from symptom onset.

\section{CONCLUSION}

The gaps in the management of Empyema in resource limited setting starts from inappropriate early treatment, inadequate diagnostic facilities, delayed referral and lack of early and appropriate surgical intervention. All these factors lead to the increased morbidity and mortality associated with the management of Empyema thoraces. These gaps need to be fulfilled by awareness at the public level, logistical up gradations at institutional level, trained manpower generation at all levels and an inclination towards early surgical intervention among the physicians.

\section{CONFLICT OF INTEREST}

None

\section{REFERENCES}

1. Weese WC, Shindler ER, Smith IM, Rabinovich S. Empyema of the thorax then and now. A study of 122 cases over four decades. Arch Intern Med. 1973 Apr;131(4):516-20. Available from: http:// www.ncbi.nlm.nih.gov/pubmed/4699956

2. Vianna NJ. Nontuberculous bacterial empyema in patients with and without underlying diseases. JAMA. 1971 Jan 4;215(1):69-75. Available from: http://www.ncbi.nlm.nih.gov/pubmed/5107351

3. Light RW. Pleural diseases. Wolters Kluwerl Lippincott Williams \& Wilkins Health; 2013. 504 p.

4. Waller DA, Rengarajan A, Nicholson FHG, Rajesh PB. Delayed referral reduces the success of video-assisted thoracoscopic debridement for post-pneumonic empyema. Respir Med. 2001 Oct;95(10):836-40. Available from: http://www. ncbi.nlm.nih.gov/pubmed/11601751

5. Banga A, Khilnani GC, Sharma SK, Dey a B, Wig N, Banga N. A study of empyema thoracis and role of intrapleural streptokinase in its management. BMC Infect Dis. 2004:4:19. Available from: http:// www.pubmedcentral.nih.gov/articlerender.fcgi?arti $d=459221 \&$ tool=pmcentrez\&rendertype=abstract

6. Baranwal AK, Singh M, Marwaha RK, Kumar L. Empyema thoracis: a 10-year comparative review of hospitalised children from south Asia. Arch Dis Child. 2003;88(11):1009-14.

7. Stern EJ, Sun H, Haramati LB. Peripheral bronchopleural fistulas: CT imaging features. 
Am J Roentgenol. 1996 Jul;167(1):117-20. Available from: http://www.ncbi.nlm.nih.gov/ pubmed/8659354

8. Ricci ZJ, Haramati LB, Rosenbaum AT, Liebling MS. Role of computed tomography in guiding the management of peripheral bronchopleural fistula. J Thorac Imaging. 2002 Jul;17(3):214-8. Available from: http://www.ncbi.nlm.nih.gov/ pubmed/12082373

9. Lois M, Noppen M. Bronchopleural Fistulas. Chest. 2005 Dec;128(6):3955-65. Available from: http:// www.ncbi.nlm.nih.gov/pubmed/16354867

10. Cerfolio RJ. The incidence, etiology, and prevention of postresectional bronchopleural fistula. Semin Thorac Cardiovasc Surg. 2001 Jan;13(1):3-7. Available from: http://www.ncbi.nlm.nih.gov/ pubmed/11309718

11. Okonta KE, Ocheli EO, Gbeneol TJ. Surgical management of recalcitrant peripheral bronchopleural fistula with empyema: A preliminary experience. Niger Med J. Medknow Publications; 2015;56(1):12-6. Available from: http://www.ncbi. nlm.nih.gov/pubmed/25657487

12. Mandal AK, Thadepalli H, Mandal AK, Chettipally $U$. Outcome of primary empyema thoracis: therapeutic and microbiologic aspects. Ann
Thorac Surg. 1998 Nov;66(5):1782-6. Available from: http://linkinghub.elsevier.com/retrieve/pii/ S0003497598006018

13. Shin JA, Chang YS, Kim TH, Haam SJ, Kim HJ, Ahn CM, et al. Surgical decortication as the firstline treatment for pleural empyema. J Thorac Cardiovasc Surg. Elsevier; 2013 Apr 1;145(4):933939.e1. Available from: http://linkinghub.elsevier. com/retrieve/pii/S0022522312008781

14. Wong KS, Lin TY, Huang YC, Chang LY, Lai $\mathrm{SH}$. Scoring system for empyema thoracis and help in management. Indian J Pediatr. 2005 Dec;72(12):1025-8. Available from: http://www. ncbi.nlm.nih.gov/pubmed/16388150

15. Thourani VH, Brady KM, Mansour KA, Miller JI, Lee RB. Evaluation of treatment modalities for thoracic empyema: a cost-effectiveness analysis. Ann Thorac Surg. 1998 Oct;66(4):1121-7. Available from: http://www.ncbi.nlm.nih.gov/ pubmed/9800792

16. Chung JH, Lee SH, Kim KT, Jung JS, Son HS, Sun K. Optimal Timing of Thoracoscopic Drainage and Decortication for Empyema. Ann Thorac Surg. 2014 Jan;97(1):224-9. Available from: http://www. ncbi.nlm.nih.gov/pubmed/24119984 